\title{
Conditional linearization of the quintic nonlinear beam equation
}

\author{
Nuntawan Sripana ${ }^{1}$ and Waraporn Chatanin $2,3^{*}$
}

\author{
"Correspondence: \\ waraporn.chat@kmutt.ac.th \\ ${ }^{2}$ Department of Mathematics, \\ Faculty of Science, King Mongkut's \\ University of Technology Thonburi, \\ Pracha-Uthit Road, Bangkok, 10140, \\ Thailand \\ ${ }^{3}$ Center of Excellence in \\ Mathematics, Bangkok, 10400 \\ Thailand \\ Full list of author information is \\ available at the end of the article
}

\begin{abstract}
In this paper, the linearization of third-order ordinary differential equations, which are the transformed equations of the quintic nonlinear beam equation, is presented. First of all, a third-order ordinary differential equation can be linearized if its coefficients satisfied the conditions of the linearization theorem. So, the conditions for linearization are investigated. After that, in each case, the linearizing transformation is defined, and finally the linear third-order equation is obtained. Moreover, after calculating the solutions to linear third-order equations and substituting the original variables to the solutions, the exact solutions to the equation of motion are obtained.
\end{abstract}

Keywords: conditional linearization; quintic nonlinear beam equation; nonlinear ordinary differential equations

\section{Introduction}

A structural element for carrying a load in buildings, bridges and steel constructions is beam. The exact solution to the problem of beam was investigated in 1927 by Love (cited in Sedighi et al. [1], p.245). Afterward, many beam problems were formulated and solved by various methods. In the case of the equation of motion for the quintic nonlinear beam, the transversal oscillation of quintic nonlinear beam was solved in the paper of Sedighi et al. [2] by using the homotopy analysis method. After that, in 2013, Sedighi et al. [1] used the stiffness analytical approximation method, the homotopy perturbation method with an auxiliary term, the max-min approach (MMA) and the iteration perturbation method (IPM) to solve the governing equation of a transversely vibrating quintic nonlinear beam. And then, the exact solution to beam vibration with quintic nonlinearity, including exact expressions for the beam curvature, was performed by using the parameter expansion method from Sedighi and Reza [3]. In 2015, Sripana and Chatanin [4] applied Lie symmetry analysis for finding the exact solutions to the nonlinear vibration of Euler-Bernoulli beam which is the equation of motion with a quintic nonlinear term. The infinitesimal generators were used to reduce the partial differential equation to the ordinary differential equation. In some cases, the ordinary differential equation could be solved analytically and give exact solution. On the other hand, some cases of nonlinear ordinary differential equations were difficult to solve analytically.

Linearization had been used to transform the nonlinear differential equations to linear equations in many fields of research. Sophus Lie [5] used the idea of point trans-

(c) The Author(s) 2017. This article is distributed under the terms of the Creative Commons Attribution 4.0 International License (http://creativecommons.org/licenses/by/4.0/), which permits unrestricted use, distribution, and reproduction in any medium, provided you give appropriate credit to the original author(s) and the source, provide a link to the Creative Commons license, and indicate if changes were made. 
formations to solve the problem on linearization of second-order ordinary differential equations. After that, the linearization of a third-order equation using the idea of point transformation was studied by Guy Grebot [6] but the problem solving in this research was not completed. In 2005, Ibragimov and Meleshko [7] presented the linearization of third-order ordinary differential equations by means of point and contact transformations. The necessary and sufficient conditions for linearization were provided in explicit forms. After that, the linearization of fourth-order ordinary differential equations by means of point transformations was studied by Ibragimov et al. [8] in 2008, and the solutions to these linearized problems were solved and presented. Moreover, a new method for reducing fourth-order autonomous ordinary differential equations (ODEs) to Lie linearizable second- and third-order ODEs was presented by Dutt and Qadir [9] in 2014.

In this paper, the linearization theorem from the research of Ibragimov and Meleshko [7] is applied for linearizing third-order ordinary differential equations. All of those equations are the transformed equations for the quintic nonlinear beam in the research of Sripana and Chatanin [4]. When the conditions for linearization in each case are investigated, linear equations are obtained. Therefore, the exact solutions to the quintic nonlinear equation of motion are obtained when all of the original variables are substituted.

\section{Review of the linearization theorem}

The necessary and sufficient conditions for linearization of the third-order ordinary differential equation by using point and contact transformations were found by Ibragimov and Meleshko in 2005 [7]. The linearization theorem states that the nonlinear ordinary differential equation, which is in the form as follows:

$$
U^{\prime \prime \prime}+\left(A_{1} U^{\prime}+A_{0}\right) U^{\prime \prime}+B_{3} U^{\prime 3}+B_{2} U^{\prime 2}+B_{1} U^{\prime}+B_{0}=0
$$

is linearizable if and only if its coefficients satisfy the following five equations:

$$
\begin{aligned}
& A_{0 U}-A_{1 z}=0, \\
& \left(3 B_{1}-A_{0}^{2}-3 A_{0 z}\right)_{U}=0, \\
& 3 A_{1 z}-A_{0} A_{1}-3 B_{2}=0, \\
& 3 A_{1 U}+A_{1}^{2}-9 B_{3}=0, \\
& \left(9 B_{1}-6 A_{0 z}-2 A_{0}^{2}\right) A_{1 z}+9\left(B_{1 z}-A_{1} B_{0}\right)_{U}+3 B_{1 U} A_{0}-27 B_{0 U U}=0 .
\end{aligned}
$$

After that, the linearizing transformations $t=\varphi(z), u=\psi(z, U)$ are defined by solving the Riccati equation

$$
\begin{aligned}
& 6 \frac{d \chi}{d z}-3 \chi^{2}=3 B_{1}-A_{0}^{2}-3 A_{0 z} \\
& \text { for } \chi=\frac{\varphi_{z z}}{\varphi_{z}}
\end{aligned}
$$


and by solving the following integrable system of partial differential equations for $\psi(z, U)$ :

$$
\begin{aligned}
& 3 \psi_{u U}=A_{1} \psi_{U}, \\
& 3 \psi_{z U}=\left(3 \chi+A_{0}\right) \psi_{U}, \\
& \psi_{z z z}=3 \chi \psi_{z z}+B_{0} \psi_{U}-\frac{1}{6}\left(3 A_{0 z}+A_{0}^{2}-3 B_{1}+9 \chi^{2}\right) \psi_{z}-\Omega \psi,
\end{aligned}
$$

where $\chi$ is given by (8). The variables are changed and a coefficient $\alpha(t)$ is found by

$$
\alpha=\Omega \varphi_{z}^{-} 3
$$

where

$$
\Omega=\frac{1}{54}\left(9 A_{0 z z}+18 A_{0 z} A_{0}+54 B_{0 U}-27 B_{1 z}+4 A_{0}^{3}-18 A_{0} B_{1}+18 A_{1} B_{0}\right) .
$$

Finally, the third-order linear equation will be obtained by

$$
u^{\prime \prime \prime}+\alpha(t) u=0
$$

\section{Equation of motion}

The partial differential equation which is the quintic nonlinear equation of motion is interesting. The nonlinear vibration of Euler-Bernoulli beam has been explained by Zhang et al. [10], whereas the governing equation which was studied by Sedighi et al. [2] is studied in this paper. A hinged-hinged flexible beam model with length subjected to constant axial load is simplified to the quintic nonlinear equation of motion. This model is studied only in the fundamental transverse mode. The interaction between transverse and longitudinal vibrations is neglected to be an assumption.

The quintic nonlinear equation of motion is in the form

$$
\begin{aligned}
& m w_{t t}+c w_{t}+E I\left[\frac{27}{2} w_{x}^{2} w_{x x}^{3}-3 w_{x x}^{3}-3 w_{x}^{2} w_{x x x x}+\frac{9}{4} w_{x}^{4} w_{x x x x}\right] \\
& +E I w_{x x x x}+P_{0} w_{x x}+\frac{3}{2} P_{0} w_{x}^{2} w_{x x}=0 .
\end{aligned}
$$

For explaining the variables and constants, the flexural deflection with respect to $x, t$ is denoted by $w$. The five important constants are a constant axial force $P_{0}$, the damping coefficient $c$, mass per unit length $m$, a modulus of elasticity $E$ and a moment of inertia $I$.

\section{Lie symmetry reduction for the quintic nonlinear beam equation}

From the research of Sripana and Chatanin [4], Lie symmetry analysis was applied to the quintic nonlinear equation of motion. The numbers of the independent variables were reduced by one, and then the partial differential equation was reduced to the ordinary 
Table 1 Two groups of nonlinear ordinary differential equations

\begin{tabular}{|c|c|c|}
\hline Group & Generators & Ordinary differential equations \\
\hline \multirow[t]{2}{*}{1} & $\begin{array}{l}X_{1}+X_{2} \\
X_{1}+x_{2}+x_{3}\end{array}$ & $\begin{array}{c}c \phi^{\prime}+\left(m+P_{0}\right) \phi^{\prime \prime}+E l \phi^{(4)}-3 E l \phi^{\prime \prime 3}+\frac{3}{2} P_{0} \phi^{\prime 2} \phi^{\prime \prime} \\
-3 E l \phi^{\prime 2} \phi^{(4)}+\frac{27}{2} E l \phi^{\prime 2} \phi^{\prime \prime 3}+\frac{9}{4} E \mid \phi^{\prime 4} \phi^{(4)}=0\end{array}$ \\
\hline & $x_{1}+x_{2}+x_{4}$ & $\begin{array}{c}c+c \phi^{\prime}+\left(m+P_{0}\right) \phi^{\prime \prime}+E l \phi^{(4)}-3 E l \phi^{\prime \prime 3}+\frac{3}{2} P_{0} \phi^{\prime 2} \phi^{\prime \prime} \\
-3 E I \phi^{\prime 2} \phi^{(4)}+\frac{27}{2} E l \phi^{\prime 2} \phi^{\prime \prime 3}+\frac{9}{4} E l \phi^{\prime 4} \phi^{(4)}=0\end{array}$ \\
\hline \multirow[t]{2}{*}{2} & $\begin{array}{l}x_{2}+x_{4} \\
x_{2}+x_{3}+x_{4}\end{array}$ & $\begin{array}{l}c+P_{0} \phi^{\prime \prime}+E l \phi^{(4)}-3 E I \phi^{\prime \prime 3}+\frac{3}{2} P_{0} \phi^{\prime 2} \phi^{\prime \prime} \\
\quad-3 E I \phi^{\prime 2} \phi^{(4)}+\frac{27}{2} E l \phi^{\prime 2} \phi^{\prime \prime 3}+\frac{9}{4} E l \phi^{\prime 4} \phi^{(4)}=0\end{array}$ \\
\hline & $x_{2}+x_{3}$ & $\begin{array}{l}P_{0} \phi^{\prime \prime}+E l \phi^{(4)}-3 E I \phi^{\prime \prime 3}+\frac{3}{2} P_{0} \phi^{\prime 2} \phi^{\prime \prime} \\
\quad-3 E I \phi^{\prime 2} \phi^{(4)}+\frac{27}{2} E \mid \phi^{\prime 2} \phi^{\prime \prime 3}+\frac{9}{4} E I \phi^{\prime 4} \phi^{(4)}=0\end{array}$ \\
\hline
\end{tabular}

Table 2 The conditions for linearization in each case

\begin{tabular}{lll}
\hline Group & Nonlinear equations & Conditions for linearization \\
\hline 1 & $E I U^{\prime \prime \prime}\left(1-3 U^{2}+\frac{9}{4} U^{4}\right)+E I U^{\prime 3}\left(\frac{27}{2} U^{2}-3\right)$ & $U=\sqrt{\frac{18}{81}}, P_{0}=0, c=0, m=0$ \\
& $+U^{\prime}\left(m+P_{0}+\frac{3}{2} P_{0} U^{2}\right)+c U=0$ & \\
& $E I U^{\prime \prime \prime}\left(1-3 U^{2}+\frac{9}{4} U^{4}\right)+E I U^{\prime 3}\left(\frac{27}{2} U^{2}-3\right)$ & $U=\sqrt{\frac{18}{81}}, P_{0}=0, c=0, m=0$ \\
& $+U^{\prime}\left(m+P_{0}+\frac{3}{2} P_{0} U^{2}\right)+c(U+1)=0$ & \\
& & \\
& $E I U^{\prime \prime \prime}\left(1-3 U^{2}+\frac{9}{4} U^{4}\right)+E I U^{\prime 3}\left(\frac{27}{2} U^{2}-3\right)$ & $U=\sqrt{\frac{18}{81}}, P_{0}=0, c=0$ \\
& $+P_{0} U^{\prime}\left(1+\frac{3}{2} U^{2}\right)+c=0$ & \\
& $E I U^{\prime \prime \prime}\left(1-3 U^{2}+\frac{9}{4} U^{4}\right)+E I U^{\prime 3}\left(\frac{27}{2} U^{2}-3\right)$ & $U=\sqrt{\frac{18}{81}}, P_{0}=0$ \\
& $+P_{0} U^{\prime}\left(1+\frac{3}{2} U^{2}\right)=0$ & \\
\hline
\end{tabular}

differential equation by using infinitesimal generators. After calculating the infinitesimal generators, four linearly independent generators were obtained as follows:

$$
\begin{array}{ll}
X_{1}=\frac{\partial}{\partial x}-\frac{m}{c} e^{-\frac{c}{m} t} \frac{\partial}{\partial w}, & X_{2}=\frac{\partial}{\partial t}-\frac{m}{c} e^{-\frac{c}{m} t} \frac{\partial}{\partial w}, \\
X_{3}=-\frac{m}{c} e^{-\frac{c}{m} t+1} \frac{\partial}{\partial w}, & X_{4}=\left[-\frac{m}{c} e^{-\frac{c}{m} t}+1\right] \frac{\partial}{\partial w} .
\end{array}
$$

The results of transforming the nonlinear partial differential equation to nonlinear ordinary differential equations are summarized in Table 1 . In Group $1, \phi^{\prime}$ is denoted by $\frac{d \phi}{d z}$ and $z=t-x$. In Group $2, z$ is denoted by $x$.

\section{Conditional linearization and exact solutions}

The conditional linearization of third-order ordinary differential equations in each case from Table 1 is presented by applying the linearization theorem of Ibragimov and Meleshko [7], and then all of nonlinear ordinary differential equations are transformed to the linear equation $u^{\prime \prime \prime}=0$. In summary, the conditions for linearization are shown in Table 2.

For finding the conditions for linearization in Group 1, the ordinary differential equation can be reduced to

$$
E I U^{\prime \prime \prime}\left(1-3 U^{2}+\frac{9}{4} U^{4}\right)+E I U^{\prime 3}\left(\frac{27}{2} U^{2}-3\right)+U^{\prime}\left(m+P_{0}+\frac{3}{2} P_{0} U^{2}\right)+c U=0,
$$

where $U^{\prime}=\frac{d U}{d z}, U=\phi^{\prime}$ and $z=t-x$. 
Table 3 The exact solutions to the quintic nonlinear equations of motion

\begin{tabular}{lll}
\hline Group & Generators & Exact solutions \\
\hline 1 & $X_{1}+X_{2}$ & $w=\frac{2 m^{2}}{c^{2}} e^{-\frac{c}{m} t}+\frac{h_{1} c_{1}^{2}}{6 b_{1}}(t-x)^{3}+\frac{h_{2} c_{1}}{2 b_{1}}(t-x)^{2}+\frac{h_{3}}{b_{1}}(t-x)+r$ \\
$X_{1}+X_{2}+X_{3}$ & $w=\frac{m^{2}}{c^{2}}(2+e) e^{-\frac{c}{m} t}+\frac{h_{1} c_{1}^{2}}{6 b_{1}}(t-x)^{3}+\frac{h_{2} c_{1}}{2 b_{1}}(t-x)^{2}+\frac{h_{3}}{b_{1}}(t-x)+r$ \\
$X_{1}+X_{2}+X_{4}$ & $w=\frac{3 m^{2}}{c^{2}} e^{-\frac{c}{m} t}+t+\frac{h_{1} c_{1}^{2}}{6 b_{1}}(t-x)^{3}+\frac{h_{2} c_{1}}{2 b_{1}}(t-x)^{2}+\frac{h_{3}}{b_{1}}(t-x)+r$ \\
2 & $X_{2}+X_{4}$ & $w=\frac{2 m^{2}}{c^{2}} e^{-\frac{c}{m} t}+t+\frac{h_{1} c_{1}^{2}}{6 b_{1}} x^{3}+\frac{h_{2} c_{1}}{2 b_{1}} x^{2}+\frac{h_{3}}{b_{1}} x+r$ \\
& $X_{2}+X_{3}+X_{4}$ & $w=\frac{m^{2}}{c^{2}}(2+e) e^{-\frac{c}{m} t}+t+\frac{h_{1} c_{1}^{2}}{6 b_{1}} x^{3}+\frac{h_{2} c_{1}}{2 b_{1}} x^{2}+\frac{h_{3}}{b_{1}} x+r$ \\
& $X_{2}+X_{3}$ & $w=\frac{m^{2}}{c^{2}}(1+e) e^{-\frac{c}{m} t}+\frac{h_{1} c_{1}^{2}}{6 b_{1}} x^{3}+\frac{h_{2} c_{1}}{2 b_{1}} x^{2}+\frac{h_{3}}{b_{1}} x+r$ \\
\hline
\end{tabular}

The coefficients of Equation (16) are specified by using the general form of Equation (1) that are $A_{0}=0, A_{1}=0, B_{2}=0, B_{0}=c U \frac{1}{E I\left(1-3 U^{2}+\frac{9}{4} U^{4}\right)}, B_{1}=\frac{\left(m+P_{0}+\frac{3}{2} P_{0} U^{2}\right)}{E I\left(1-3 U^{2}+\frac{9}{4} U^{4}\right)}$, and $B_{3}=\frac{\left(\frac{27}{2} U^{2}-3\right)}{\left(1-3 U^{2}+\frac{9}{4} U^{4}\right)}$. From the necessary conditions, conditions (2) and (4) are satisfied. Condition (3), that is, $\frac{d}{d U}\left[\frac{\left(3 m+3 P_{0}+\frac{9}{2} P_{0} U^{2}\right)}{E I\left(1-3 U^{2}+\frac{9}{4} U^{4}\right)}\right]=0$, will be satisfied if and only if $U=0$ or $m, P_{0}=0$. Condition (5), that is, $-9\left[\frac{\left(\frac{27}{2} U^{2}-3\right)}{\left(1-3 U^{2}+\frac{9}{4} U^{4}\right)}\right]=0$, will be satisfied if and only if $U=\sqrt{\frac{18}{81}}$. Condition (6), that is, $-27 \frac{d^{2}}{d U^{2}}\left[c U \frac{1}{E I\left(1-3 U^{2}+\frac{9}{4} U^{4}\right)}\right]=0$, will be satisfied if and only if $U=0$ or $c=0$. In the same way, the conditions for linearization in Group 2 are satisfied by letting $U=\sqrt{\frac{18}{81}}$ and $P_{0}=0$. By comparing the coefficients with Equation (1), we have $A_{0}=0$, $A_{1}=0, B_{0}=0, B_{1}=0, B_{2}=0$ and $B_{3}=\frac{\left(\frac{27}{2} U^{2}-3\right)}{\left(1-3 U^{2}+\frac{9}{4} U^{4}\right)}$.

The linearizing transformation $\zeta=\varphi(z)$ is defined by solving the Riccati equation $6 \frac{d \chi}{d z}-3 \chi^{2}=0$, and the simplest solution is $\chi=0$. By considering the equation $\chi=\frac{\varphi_{z z}}{\varphi_{z}}$, the simplest solution for $\varphi(z)$ is $\varphi=c_{1} z$. For finding $\psi(z, U)$, we get $3 \psi_{U U}=0$ and $3 \psi_{z U}=0$. The simplest solution for $\psi(z, U)$ is $\psi=b_{1} U$. So, the linearizing transformation is $\zeta=c_{1} z$ and $u=b_{1} U$. The coefficient $\alpha(\zeta)$ is found by using $\alpha=\Omega \varphi_{z}^{-3}$, where $\Omega=\frac{1}{54}\left(9 A_{0 z z}+18 A_{0 z} A_{0}+54 B_{0 U}-27 B_{1 z}+4 A_{0}^{3}-18 A_{0} B_{1}+18 A_{1} B_{0}\right)$. By calculating $\Omega=0$ and $\varphi_{z}^{-3}=c_{1}^{-3}$, the coefficient $\alpha(\zeta)$ is equal to zero. Therefore, the third-order linear equation $u^{\prime \prime \prime}+\alpha(\zeta) u=0$ becomes $u^{\prime \prime \prime}=0$. After calculating the solution to this third-order linear equation, we get $u=h_{1} \frac{\zeta^{2}}{2}+h_{2} \zeta+h_{3}$, that is, $U=h_{1} \frac{\left(c_{1} z\right)^{2}}{2 b_{1}}+h_{2} \frac{\left(c_{1} z\right)}{b_{1}}+\frac{h_{3}}{b_{1}}$. After that, the original variable $U=\phi^{\prime}$ is substituted into the solution, which gives $\phi=\frac{h_{1} c_{1}^{2}}{6 b_{1}} z^{3}+\frac{h_{2} c_{1}}{2 b_{1}} z^{2}+\frac{h_{3}}{b_{1}} z+r$. Finally, all of solutions are obtained by substituting the original variables in each case as shown in Table 3.

\section{Conclusions}

In this research, the conditional linearization of third-order ordinary differential equations is presented. The transformed equations of the quintic nonlinear beam equation in the research of Sripana and Chatanin, which are fourth-order nonlinear ordinary differential equations, can be reduced to third-order ordinary differential equations and have the form that corresponds to the third-order equation in the linearization theorem of Ibagimov and Meleshko. So the linearization theorem is applied for linearizing these equations. First, the linearization conditions are considered. Then, the linearizing transformation is defined. Finally, the linear third-order differential equations which can be solved analytically are 
obtained. Furthermore, the exact solutions to the quintic nonlinear beam equation are also obtained after substituting all the original variables to the solutions.

\section{Competing interests}

The authors declare that they have no competing interests.

\section{Authors' contributions}

All authors contributed equally to the writing of this paper. All authors read and approved the final manuscript.

\section{Author details}

'Faculty of Liberal Arts, Rajamangala University of Technology Tawan-Ok Chakrabongse Bhuvanarth Campus, Vibhavadi Rangsit Road, Bangkok, 10400, Thailand. ${ }^{2}$ Department of Mathematics, Faculty of Science, King Mongkut's University of Technology Thonburi, Pracha-Uthit Road, Bangkok, 10140, Thailand. ${ }^{3}$ Center of Excellence in Mathematics, Bangkok, 10400, Thailand.

\section{Acknowledgements}

This work was supported by Rajamangala University of Technology Tawan-Ok Chakrabongse Bhuvanarth Campus, Department of Mathematics, Faculty of Science, King Mongkut's University of Technology Thonburi, Thailand and the Center of Excellence in Mathematics.

\section{Publisher's Note}

Springer Nature remains neutral with regard to jurisdictional claims in published maps and institutional affiliations.

Received: 2 February 2017 Accepted: 11 April 2017 Published online: 21 April 2017

\section{References}

1. Sedighi, MH, Shirazi, HK, Attarzadeh, AM: A study on the quintic nonlinear beam vibrations using asymptotic approximate approaches. Acta Astronaut. 91, 245-250 (2013)

2. Sedighi, MH, Shirazi, HK, Zare, J: An analytic solution of transversal oscillation of quintic non-linear beam with homotopy analysis method. Int. J. Non-Linear Mech. 47, 777-784 (2012)

3. Sedighi, MH, Reza, A: High precise analysis of lateral vibration of quintic nonlinear beam. Lat. Am. J. Solids Struct. 10 441-452 (2013)

4. Sripana, N, Chatanin, W: Similarity reductions for the nonlinear vibration of Euler-Bernoulli beam. In: Proceedings of Ramkhamhaeng University International Research Conference 2015 Bangkok, 2-3 September 2015, pp. 178-185. Research and Development Institute, Ramkhamhaeng University (2015)

5. Lie, S: Klassifikation und integration von gewohnlichen differentialgleichungen zwischen $x, y$, die eine gruppe von transformationen gestatten iii. Arch. Math. Naturvidensk. 8, 371-458 (1883)

6. Grebot, G: The characterization of third order ordinary differential equations admitting a transitive fiber-preserving point symmetry group. J. Math. Anal. Appl. 206, 364-388 (1997)

7. Ibragimov, NH, Meleshko, SV: Linearization of third-order ordinary differential equations by point and contact transformations. J. Math. Anal. Appl. 308, 266-289 (2005)

8. Ibragimov, NH, Meleshko, SV, Suksern, S: Linearization of fourth-order ordinary differential equations by point transformations. J. Phys. A, Math. Theor. 41, 235206 (2008)

9. Dutt, $\mathrm{MH}$, Qadir, A: Reduction of fourth order ordinary differential equations to second and third order lie linearizable forms. Commun. Nonlinear Sci. Numer. Simul. 19, 2653-2659 (2014)

10. Zhang, W, Wang, FX, Zu, JW: Local bifurcations and codimension-3 degenerate bifurcations of a quintic nonlinear beam under parametric excitation. Chaos Solitons Fractals 24, 977-998 (2005)

\section{Submit your manuscript to a SpringerOpen ${ }^{\circ}$ journal and benefit from:}

- Convenient online submission

Rigorous peer review

- Immediate publication on acceptance

- Open access: articles freely available online

- High visibility within the field

- Retaining the copyright to your article 